\title{
Neural G protein-coupled receptor OCTR-1 mediates temperature effects on longevity by regulating immune response genes in C. elegans
}

Shawndra Wibisono ${ }^{1}$, Phillip Wibisono ${ }^{1}$, Jingru Sun ${ }^{1 *}$

${ }^{1}$ Department of Biomedical Sciences, Elson S. Floyd College of Medicine, Washington State University, Spokane, WA, USA

*To whom correspondence should be addressed: jingru.sun@wsu.edu

\author{
ABSTRACT \\ We have previously demonstrated that OCTR-1, an octopamine G protein-couple receptor, \\ functions in the sensory neurons $\mathrm{ASH}$ to suppress the innate immune response in \\ Caenorhabditis elegans by inhibiting the expression of immune genes (Sun et al. 2011 Science \\ 332:729-732). Here we discover that OCTR-1 also regulates temperature effects on lifespan in \\ C. elegans. At the normal growth temperature $20^{\circ} \mathrm{C}$, octr-1(ok371) mutant animals have similar \\ lifespan to wild-type $\mathrm{N} 2$ animals. However, at higher temperature $25^{\circ} \mathrm{C}$, octr-1(ok371) mutants \\ live significant longer than wild-type N2 animals. These results suggest that OCTR-1 may \\ mediate temperature effects on lifespan. Furthermore, we found the OCTR-1-expressing ASH \\ chemosensory neurons are involved in the OCTR-1-mediated regulation on longevity. However, \\ interestingly, the thermosensory AFD neurons do not play a role in this regulation at $25^{\circ} \mathrm{C}$. RNA- \\ seq data analysis showed that 63 immune response genes were significantly down-regulated in \\ octr-1(ok371) mutants relative to wild-type animals at $25^{\circ} \mathrm{C}$. We further demonstrated that \\ inactivation of several most-downregulated genes by RNA interference in wild-type N2 animals \\ significantly extended their lifespan, similar to the phenotype of octr-1(ok371) animals. These \\ observations suggest a new molecular regulation mechanism that downregulation of immune
}


genes extends the lifespan of $C$. elegans, which is opposite to the general belief that an increase in defense immunity extends lifespan.

Key words: Caenorhabditis elegans, immune response genes, G protein-coupled receptor, temperature effect, longevity, thermosensory neurons, chemosensory neurons.

\section{INTRODUCTION}

Various studies in both poikilotherms and homeotherms have associated lower temperature with longer lifespan and higher temperature with shorter lifespan (1). These inverse effects of temperature on longevity were traditionally explained by the "rate of living theory", which posits that lower temperatures reduce chemical reaction rates, thus slowing down the aging process, whereas higher temperatures do the opposite (2). Recent studies have identified specific molecules and cells that mediate the longevity responses to temperature, suggesting that the temperature effects on aging are not simply thermodynamic but regulated processes (3-5). The mechanisms underlying such regulation, however, are not well understood. Caenorhabditis elegans is one of the organisms that were used in early studies of the temperature effects on longevity, and a negative correlation has been well documented (6). We have discovered that $C$. elegans lacking OCTR-1, a neuronal G protein-coupled receptor (GPCR) for the neurotransmitter octopamine $(\mathrm{OA})(7)$, exhibited increased lifespan at warm temperature $\left(25^{\circ} \mathrm{C}\right)$ relative to wild-type N2 animals, indicating a critical role of OCTR-1 in modulating the temperature effects on longevity/aging. This modulation involves the OCTR-1-expressing sensory neurons ASH but surprisingly not the thermosensory neurons AFD. Further RNA-seq data analysis showed that 63 immune defense genes were significantly down-regulated in octr1(ok371) mutants relative to wild-type animals at $25^{\circ} \mathrm{C}$. Moreover, we demonstrated that inactivation of several most-downregulated genes by RNA interference (RNAi) in wild-type 
animals significantly extended their lifespan, similar to the phenotype of octr-1(ok371) animals. These suggest a new molecular regulation mechanism that downregulation of immune genes extend the lifespan of $C$. elegans, which is opposite to the general belief that an increase in defense immunity extends lifespan.

\section{RESULTS AND DISCUSSION}

octr-1(ok371) mutant animals live significantly longer than wild-type animals at high temperature

We have demonstrated that the OCTR-1 neural circuit controls the survivals of $C$. elegans against pathogen infection by suppressing the unfolded protein response (UPR) pathways (8-9). To test whether such regulation of lifespan also occurs under other stress conditions, such as higher growth temperature, we compared the lifespans of wild-type N2 animals and octr1(ok371) mutant animals at $20^{\circ} \mathrm{C}$ (standard growth temperature) and $25^{\circ} \mathrm{C}$ (higher growth temperature). As shown in Figure 1, the octr-1(ok371) mutant animals live similar to wild-type $\mathrm{N} 2$ animals at $20^{\circ} \mathrm{C}$ (Fig. $\left.1 \mathrm{~A}\right)$, but live significantly longer than the $\mathrm{N} 2$ animals at $25^{\circ} \mathrm{C}$ (Fig. $1 \mathrm{~B}$ ). These results suggest that OCTR-1 neural circuit may mediate temperature effects on lifespan at higher temperature.

\section{The ASH chemosensory neurons, but not the AFD thermosensory neurons, are involved in the OCTR-1 neural circuit to regulate longevity at high temperature}

We have previously demonstrated that OCTR-1 functions in the chemosensory neurons ASH to suppress the innate immune response in $C$. elegans by inhibiting the expression of immune genes (8). To determine if ASH neurons are involved in the OCTR-1 neural circuit-regulated longevity at high temperature, we examined the lifespans of $\mathrm{ASH}$ mutants and $\mathrm{ASH}$;octr1(ok371) mutants at $25^{\circ} \mathrm{C}$. As shown in Figure 2A, both ASH mutants and ASH;octr-1(ok371) mutants live significantly longer than wild-type $\mathrm{N} 2$ animals at $25^{\circ} \mathrm{C}$, suggesting that the ASH 
chemosensory neurons play an important role in the OCTR-1-dependent regulation of longevity at high temperature.

C. elegans senses temperature using the AFD thermosensory neurons. The thermosensory circuit for response to temperature changes has been well studied (10). To determine if the AFD thermosensory neurons are involved in the OCTR-1 neural circuit-regulated longevity at high temperature, we examined the lifespans of AFD mutants $(t t x-1(p 767))$ and $t t x-1(p 767)$;octr1(ok371) mutants at $25^{\circ} \mathrm{C}$. As shown in Figure 2B, the $t$ tx-1(p767);octr-1(ok371) mutants live significantly longer than wild-type $\mathrm{N} 2$ animals at $25^{\circ} \mathrm{C}$, but no difference in lifespan was observed between AFD mutants and wild-type N2 animals. These results suggest that the AFD thermosensory neurons do not play an important role in the OCTR-1-dependent regulation of longevity at high temperature. The role of the AFD neurons in sensing higher temperature at $25^{\circ} \mathrm{C}$ might be counterbalanced by an unidentified neuron(s) in the OCTR-1 neural circuit, which grants a future investigation.

\section{Immune defense genes are significantly down-regulated in octr-1(ok371) mutants at high temperature}

To gain insights on how the OCTR-1 neural circuit mediates temperature effects on longevity at the molecular level, we used RNA-seq to profile gene expression in octr-1(ok371) animals relative to wild-type $\mathrm{N} 2$ animals at $20^{\circ} \mathrm{C}$ and $25^{\circ} \mathrm{C}$. To this end, we collected five replicates of four groups of RNA samples [octr-1(ok371)] and wild-type N2 animals grown on E. coli OP50 for 14 days (TD50) at $20^{\circ} \mathrm{C}$ or 9 days (TD50) at $25^{\circ} \mathrm{C}$. These samples were submitted to the Genomics Core for RNA-seq analysis. The resulting sequence data will be deposited in the National Center for Biotechnology Information's (NCBI) Sequence Read Archive (SRA) database through the Gene Expression Omnibus (GEO); processed gene quantification files and differential expression files will be deposited in GEO. 
To examine how OCTR-1 neural circuit regulates gene expression in C. elegans at high temperature, we compared the expression profile of octr-1(ok371) animals with that of wild-type $\mathrm{N} 2$ animals at $25^{\circ} \mathrm{C}$. We found that 912 genes were down-regulated at least twofold in the mutant animals. Ontology analysis of the down-regulated genes revealed one significantly enriched molecular function involved in immune defense response. The top 10 most downregulated immune genes among the 63 defense genes are listed in Table 1 . These results indicate that down-regulation of immune defense genes by the OCTR-1 neural circuit could play a role in extending the lifespan of $C$. elegans at high temperature.

\section{Inactivation of the most down-regulated genes by RNA interference in wild-type animals extends their lifespans}

To examine whether the OCTR-1-downregulated immune defense genes contribute to the improved lifespan of the mutant animals, we inactivated these genes by RNA interference (RNAi) in wild-type $\mathrm{N} 2$ animals and assayed their lifespans at $25^{\circ} \mathrm{C}$. As demonstrated in Figure 3 , inactivation of the lys-5, acdh-1, lys-4, and srx-128 genes significantly enhanced their lifespans, similar to the lifespan of octr-1(ok371) mutant animals at $25^{\circ} \mathrm{C}$, indicating that the OCTR-1-downregulated immune defense genes contribute to the enhanced lifespan of octr1(ok371) animals at $25^{\circ} \mathrm{C}$. These observations suggest a new molecular regulation mechanism that downregulation of immune genes extends the lifespan of $C$. elegans, which is opposite to the general belief that an increase in defense immunity extends lifespan. It would be interesting to investigating how the lifespan of $C$. elegans is enhanced by downregulating the immune defense genes.

MATERIALS AND METHODS

\section{Nematode strains}


The following C. elegans strains were cultured under standard conditions and fed Escherichia coli OP50. Wild-type N2 were C. elegans Bristol N2. The octr-1(ok371), ttx-1(p767), and ASH(-) [JN1713] strains were obtained from the Caenorhabditis elegans Genetics Center (University of Minnesota, Minneapolis, MN). The ASH(-);octr-1(ok371) and ttx-1(p767);octr-1(ok371) mutant strains were constructed using standard genetic techniques.

\section{Bacterial strain}

E. coli strain OP50 were grown in Luria-Bertani (LB) broth at $37^{\circ} \mathrm{C}$.

\section{Lifespan assay}

C. elegans wild-type N2 animals and mutant animals were maintained as hermaphrodites at $20^{\circ} \mathrm{C}$, grown on modified nematode growth medium (NGM) agar plates $(0.35 \%$ instead of $0.25 \%$ peptone). Bacterial lawns used for the lifespan assays were prepared by placing a $50 \mu \mathrm{L}$ drop of an overnight fresh culture of E. coli OP50 on each $3.5 \mathrm{~cm}$ plate. Plates were incubated at $37^{\circ} \mathrm{C}$ for 16 hours, cooled down to room temperature, and then seeded with synchronized 65-hour-old young adult animals. These worms were prepared by undergoing two rounds of egg-laying synchronization before they were exposed to E. coli OP50 for lifespan assays. The lifespan assays were performed at $20^{\circ} \mathrm{C}$ or $25^{\circ} \mathrm{C}$. Live animals were transferred daily to fresh plates. Animals were scored at the times indicated and were considered dead when they failed to respond to touch.

\section{RNA sequencing}

Five replicates of four groups of RNA samples [octr-1(ok371)] and wild-type N2 animals grown on E. coli OP50 for 14 days at $20^{\circ} \mathrm{C}$ or 9 days at $25^{\circ} \mathrm{C}$ were collected and submitted to the Genomics Core for RNA-seq analysis. The integrity of total RNA was assessed using Fragment Analyzer (Advanced Analytical Technologies, Ankeny, IA) with the High Sensitivity RNA 
Analysis Kit. RNA quality numbers (RQNs) from 1 to 10 was assigned to each sample to indicate its integrity or quality. "10" stands for a perfect RNA sample without any degradation, whereas "1" marks a completely degraded sample. RNA samples with RQNs ranging from 8 to 10 were used for RNA library preparation with the TruSeq Stranded mRNA Library Prep Kit (Illumina, San Diego, CA). Briefly, mRNA was isolated from $2.5 \mu \mathrm{g}$ of total RNA using poly-T oligo attached to magnetic beads and then subjected to fragmentation, followed by cDNA synthesis, dA-tailing, adaptor ligation and PCR enrichment. The sizes of the RNA libraries were assessed by Fragment Analyzer with the High Sensitivity NGS Fragment Analysis Kit. The concentrations of the RNA libraries were measured using a StepOnePlus Real-Time PCR System (ThermoFisher Scientific, San Jose, CA) with the KAPA Library Quantification Kit (Kapabiosystems, Wilmington, MA). The libraries were diluted to $2 \mathrm{nM}$ with RSB (10 mM Tris$\mathrm{HCl}, \mathrm{pH} 8.5$ ) and denatured with $0.1 \mathrm{~N} \mathrm{NaOH}$. Eighteen $\mathrm{pM}$ libraries were clustered in a highoutput flow cell using HiSeq Cluster Kit v4 on a cBot (Illumina). After cluster generation, the flow cell was loaded onto a HiSeq 2500 for sequencing using a HiSeq SBS kit v4 (Illumina). DNA was sequenced from both ends (paired-end) with a read length of $100 \mathrm{bp}$. The raw BCL files were converted to FASTQ files using the software program bcl2fastq2.17.1.14. Adaptors were trimmed from the FASTQ files during the conversion. On average, 40 million reads with a read length of 2x 100 bp were generated for each sample. RNA-seq data (FASTQ files) were aligned to the C. elegans reference genome (ce10, UCSC) using HISAT2. Gene expression quantification and differential expression were analyzed using HTSeq and DESeq2.

\section{RNA interference}

RNAi was conducted by feeding L2 or L3 larval C. elegans E. coli strain HT115(DE3) expressing double-stranded RNA (dsRNA) that was homologous to a target gene (11). E. coli with the appropriate dsRNA vector were grown in LB broth containing ampicillin $(100 \mu \mathrm{g} / \mathrm{mL})$ at $37^{\circ} \mathrm{C}$ for 16 hours and plated on modified NGM plates containing $100 \mu \mathrm{g} / \mathrm{mL}$ ampicillin and $3 \mathrm{mM}$ 
isopropyl $\beta$-D-thiogalactoside (IPTG). The RNAi-expressing bacteria were allowed to grow for 16 hours at $37^{\circ} \mathrm{C}$. The plates were cooled down before the L2 or L3 larval animals were placed on the bacteria. The animals were incubated at $20^{\circ} \mathrm{C}$ for 24 hours or until the animals were 65 hours old. unc-22 RNAi was included as a positive control in all experiments to account for RNAi efficiency. Clone identity was confirmed by sequencing at Eton Bioscience Inc. (San Diego, CA).

\section{Statistical analysis}

Lifespan curves were plotted using GraphPad PRISM (version 9) computer software. Lifespan was considered different from the appropriate control indicated in the main text when $P<0.05$. PRISM uses the product limit or Kaplan-Meier method to calculate survival fractions and the logrank test, which is equivalent to the Mantel-Haenszel test, to compare survival curves. All experiments were repeated at least three times, unless otherwise indicated.

\section{ACKNOWLEDGEMENTS}

We would like to thank the Caenorhabditis Genetics Center (CGC) for providing several worm strains used in this study. CGC is funded by the NIH Office of Research Infrastructure Programs (P40 OD010440).

\section{FUNDING}

This work was supported by NIH (R35GM124678 to J.S.). The funder had no role in study design, data collection and interpretation, or the decision to submit the work for publication.

\section{AUTHOR CONTRIBUTIONS}


S.W., P.W., and J.S. designed and performed experiments and analyzed data. J.S. wrote the paper.

\section{COMPETING INTERESTS}

The authors declare that they have no competing interests.

\section{DATA AND MATERIALS AVAILABILITY}

The RNA-seq data will be deposited in NCBI's SRA database through the GEO. The processed gene quantification files and differential expression files will be deposited in the GEO. All data needed to evaluate the conclusions in the paper are present in the paper. Additional data related to this paper may be requested from the authors. The $C$. elegans strains and plasmids constructed by us and primer sequences used for their construction are available upon request.

\section{REFERENCES}

1. Keil, G., Cummings, E., and de Magalhaes, J. P. (2015) Being cool: how body temperature influences ageing and longevity. Biogerontology 16, 383-397

2. Demetrius, L. A. (2013) Boltzmann, Darwin and Directionality theory. Physics Reports $530,1-85$

3. Xiao, R., Zhang, B., Dong, Y., Gong, J., Xu, T., Liu, J., and Xu, X. Z. (2013) A genetic program promotes C. elegans longevity at cold temperatures via a thermosensitive TRP channel. Cell 152, 806-817

4. Lee, S. J., and Kenyon, C. (2009) Regulation of the longevity response to temperature by thermosensory neurons in Caenorhabditis elegans. Current biology : $C B$ 19, 715-722 
5. Conti, B., Sanchez-Alavez, M., Winsky-Sommerer, R., Morale, M. C., Lucero, J., Brownell, S., Fabre, V., Huitron-Resendiz, S., Henriksen, S., Zorrilla, E. P., de Lecea, L., and Bartfai, T. (2006) Transgenic mice with a reduced core body temperature have an increased life span. Science 314, 825-828

6. Van Voorhies, W. A., and Ward, S. (1999) Genetic and environmental conditions that increase longevity in Caenorhabditis elegans decrease metabolic rate. Proceedings of the National Academy of Sciences of the United States of America 96, 11399-11403

7. Sellegounder, D., Yuan, C. H., Wibisono, P., Liu, Y., and Sun, J. (2018) Octopaminergic Signaling Mediates Neural Regulation of Innate Immunity in Caenorhabditis elegans.

\section{MBio 9}

8. Sun, J., Singh, V., Kajino-Sakamoto, R., and Aballay, A. (2011) Neuronal GPCR controls innate immunity by regulating noncanonical unfolded protein response genes. Science

\section{$332,729-732$}

9. Sun, J., Liu, Y., and Aballay, A. (2012) Organismal regulation of XBP-1-mediated unfolded protein response during development and immune activation. EMBO reports $13,855-860$

10. Mori, I., and Ohshima, Y. (1995) Neural regulation of thermotaxis in Caenorhabditis elegans. Nature 376, 344-348

11. Timmons, L., and Fire, A. (1998) Specific interference by ingested dsRNA. Nature 395, 854

\section{FIGURE LEGENDS}

Fig 1. The lifespan of octr-1(ok371) mutants is similar to that of wild-type N2 animals at $20^{\circ} \mathrm{C}$ but significantly longer than $\mathrm{N} 2$ at $25^{\circ} \mathrm{C}$. Wild-type $\mathrm{N} 2$ and octr-1(ok371) mutant animals were grown on C. elegans laboratory food E. coli OP50 and scored for lifespan over 
time at $20^{\circ} \mathrm{C}$ and $25^{\circ} \mathrm{C}$, respectively. The graphs are the representative results of three independent experiments. Each experiment included $n=90$ adult animals per strain. $P$ value represents the significance level of the mutants relative to the wild-type N2: $p=0.8410$ for $(A)$ and $p=0.0018$ for $(\mathrm{B})$.

Fig. 2. The ASH neurons, but not the AFD neurons, play an important role in the OCTR-1dependent regulation of longevity at $25^{\circ} \mathrm{C}$. (A) Wild-type N2, octr-1(ok371), ASH(-), and ASH(-);octr-1(ok371) mutant animals were grown on C. elegans laboratory food E. coli OP50 and scored for lifespan over time at $25^{\circ} \mathrm{C}$. The graph is a representative result of three independent experiments. Each experiment included $n=90$ adult animals per strain. $P$ value represents the significance level of the mutants relative to the wild-type N2: octr-1(ok371), $p<0.0001 ; \mathrm{ASH}(-), p=0.0008 ; \mathrm{ASH}(-) ;$ octr-1(ok371), $p<0.0001$. (B) Wild-type N2, octr-1(ok371), $t t x-1(p 767)$, and $t t x-1$ (p767);octr-1(ok371) mutant animals were grown on $C$. elegans laboratory food $E$. coli OP50 and scored for lifespan over time at $25^{\circ} \mathrm{C}$. The graph is a representative result of three independent experiments. Each experiment included $\mathrm{n}=90$ adult animals per strain. $P$ value represents the significance level of the mutants relative to the wild-type N2: octr-1(ok371), $p<0.0001 ; t t x-1(p 767), p=0.1862 ; t t x-1(p 767) ; o c t r-1$ (ok371), $p<0.0001$.

Fig 3. The OCTR-1-downregulated immune defense genes contribute to the improved lifespan of octr-1(ok371) animals at $25^{\circ} \mathrm{C}$. Wild-type $\mathrm{N} 2$ and octr-1(ok371) animals grown on dsRNA for several most-downregulated genes or empty vector (EV) control were exposed to $C$. elegans laboratory food E. coli OP50 and scored for lifespan over time at $25^{\circ} \mathrm{C}$. The graphs are the combined results of three independent experiments. Each experiment included $n=60$ adult animals per strain. $P$ value represents the significance level of the mutants relative to the wildtype N2+EV: (A) octr-1(ok371)+EV, p<0.0001; N2+lys-5 RNAi, p=0.0007; (B) N2+acdh-1 RNAi, $p<0.0001$; (C) N2+lys-4 RNAi, $p=0.0296$; (D) N2+srx-128 RNAi, $p=0.0001$. 
Fig. 1

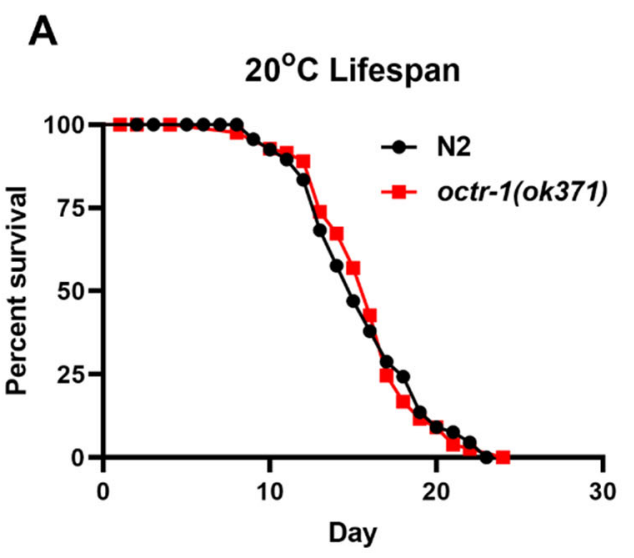

B

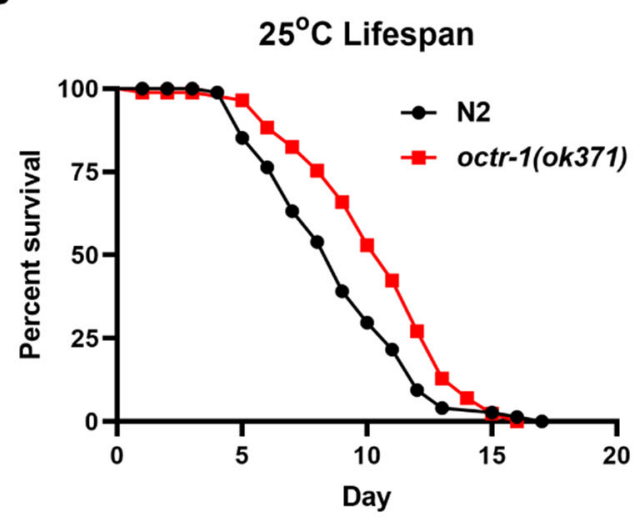

Fig. 1. The lifespan of octr-1(ok371) mutants is similar to that of wild-type N2 animals at $20^{\circ} \mathrm{C}$ but significantly longer than $\mathrm{N} 2$ at $25^{\circ} \mathrm{C}$. Wild-type $\mathrm{N} 2$ and octr-1(ok371) mutant animals were grown on C. elegans laboratory food E. coli OP50 and scored for lifespan over time at $20^{\circ} \mathrm{C}$ and $25^{\circ} \mathrm{C}$, respectively. The graphs are the representative results of three independent experiments. Each experiment included $n=90$ adult animals per strain. $P$ value represents the significance level of the mutants relative to the wild-type N2: $p=0.8410$ for $(A)$ and $p=0.0018$ for $(\mathrm{B})$. 
Fig. 2.
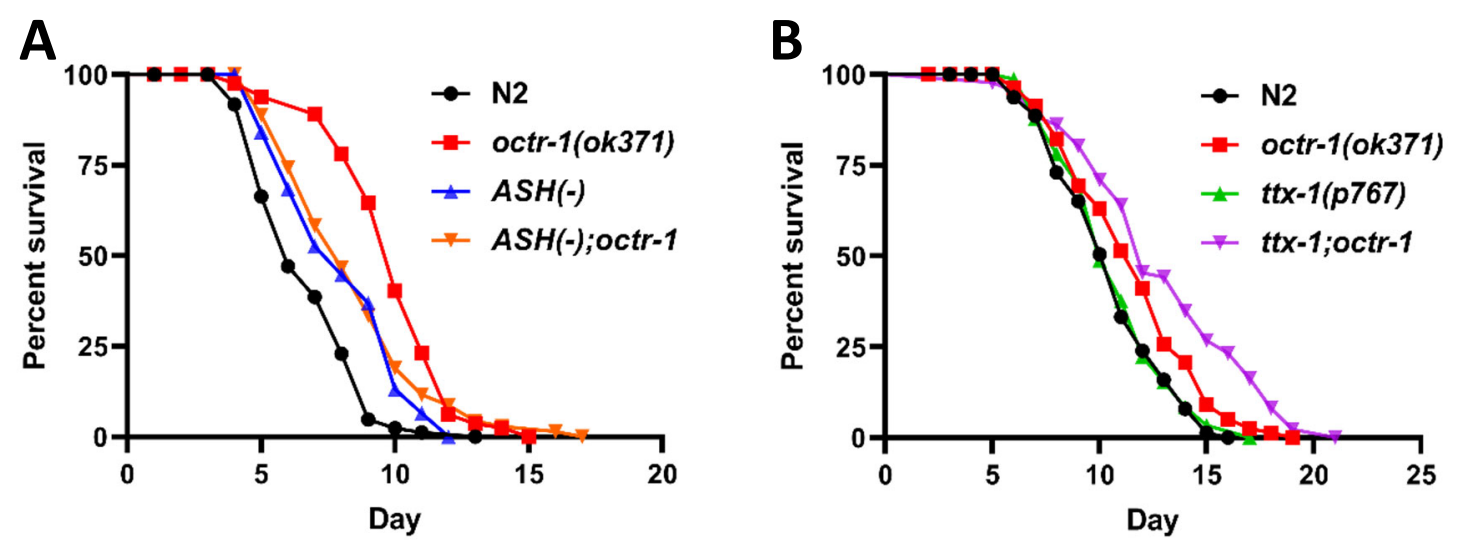

Fig. 2. The ASH neurons, but not the AFD neurons, play an important role in the OCTR-1dependent regulation of longevity at $25^{\circ} \mathrm{C}$. (A) Wild-type $\mathrm{N} 2$, octr-1(ok371), $A S H(-)$, and ASH(-);octr-1(ok371) mutant animals were grown on C. elegans laboratory food E. coli OP50 and scored for lifespan over time at $25^{\circ} \mathrm{C}$. The graph is a representative result of three independent experiments. Each experiment included $n=90$ adult animals per strain. $P$ value represents the significance level of the mutants relative to the wild-type N2: octr-1(ok371), $p<0.0001 ; \mathrm{ASH}(-), p=0.0008 ; \mathrm{ASH}(-)$;octr-1(ok371), $p<0.0001$. (B) Wild-type N2, octr-1(ok371), ttx-1(p767), and ttx-1(p767);octr-1(ok371) mutant animals were grown on C. elegans laboratory food $E$. coli OP50 and scored for lifespan over time at $25^{\circ} \mathrm{C}$. The graph is a representative result of three independent experiments. Each experiment included $\mathrm{n}=90$ adult animals per strain. $P$ value represents the significance level of the mutants relative to the wild-type N2: octr-1(ok371), $p<0.0001 ; t t x-1(p 767), p=0.1862 ; t t x-1(p 767) ; o c t r-1$ (ok371), $p<0.0001$. 
Fig. 3

A

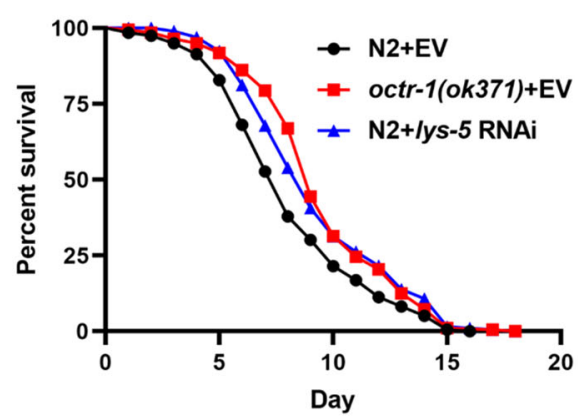

C

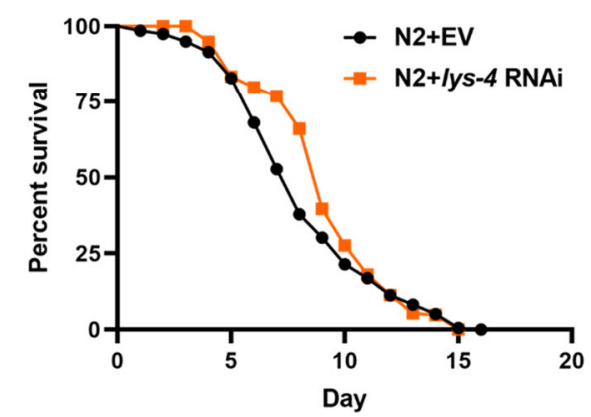

B

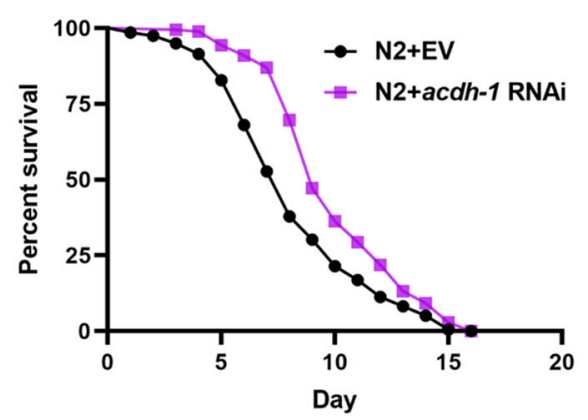

D

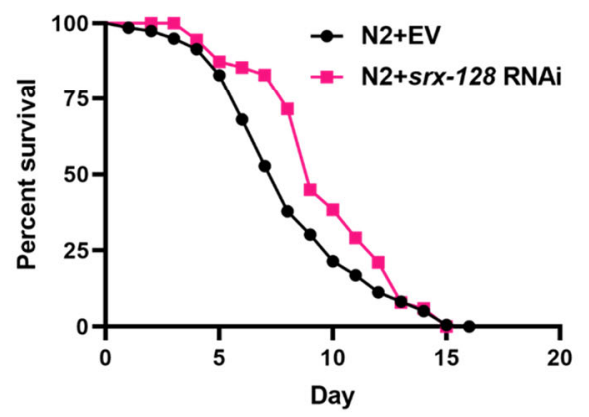

Fig. 3. The OCTR-1-downregulated immune defense genes contribute to the improved lifespan of octr-1(ok371) animals at $25^{\circ} \mathrm{C}$. Wild-type $\mathrm{N} 2$ and octr-1(ok371) animals grown on dsRNA for several most-downregulated genes or empty vector (EV) control were exposed to $C$. elegans laboratory food E. coli OP50 and scored for lifespan over time at $25^{\circ} \mathrm{C}$. The graphs are the combined results of three independent experiments. Each experiment included $n=60$ adult animals per strain. $P$ value represents the significance level of the mutants relative to the wildtype N2+EV: (A) octr-1(ok371)+EV, $p<0.0001$; N2+lys-5 RNAi, $p=0.0007$; (B) N2+acdh-1 RNAi, $p<0.0001$; (C) N2+lys-4 RNAi, $p=0.0296$; (D) N2+srx-128 RNAi, $p=0.0001$. 
Table 1. Top 10 OCTR-1-downregulated immune defense genes at $25^{\circ} \mathrm{C}$.

\begin{tabular}{|l|l|l|}
\hline \multicolumn{3}{|l|}{ Top 10 down-regulated immune genes in N2_25'C vs octr-1_25 ${ }^{\circ}$ C } \\
\hline Sequence name & Gene name & Fold_down \\
\hline F58B3.2 & lys-5 & 146.9 \\
\hline C55B7.4 & acdh-1 & 63.7 \\
\hline C34F6.3 & col-179 & 41 \\
\hline F58B3.1 & lys-4 & 26.2 \\
\hline F22A3.6 & ilys-5 & 23.4 \\
\hline K12H4.7 & & 15.7 \\
\hline Y22F5A.5 & lys-2 & 14.1 \\
\hline T08A9.7 & spp-3 & 12.6 \\
\hline K08D8.5 & & 12.5 \\
\hline C02A12.4 & lys-7 & 11.6 \\
\hline
\end{tabular}

